\title{
Rapid Fenton-like degradation of methyl orange by ultrasonically dispersed nano-metallic particles
}

\author{
Jiwan Singh $^{1^{\dagger}}$, Yoon-Young Chang ${ }^{2}$, Janardhan Reddy Koduru ${ }^{{ }^{\dagger}}$, Jae-Kyu Yang ${ }^{3}$, Devendra Pratap Singh ${ }^{1}$ \\ ${ }^{1}$ Department of Environmental Science, Babasaheb Bhimrao Ambedkar University, Lucknow-226025, India \\ ${ }^{2}$ Department of Environmental Engineering, Kwangwoon University, Seoul 01897, Republic of Korea \\ ${ }^{3}$ Ingenium College of Liberal Arts, Kwangwoon University, Seoul 01897, Republic of Korea
}

\begin{abstract}
This study investigates methyl orange (MO) degradation by an ultrasonically dispersed nano-metallic particle (NMP) assisted advanced Fenton process. The NMPs were synthesized from the leachate of automobile-shredder residue. X-ray photoelectron spectroscopy and Fourier-transform infrared spectroscopy were performed for the prepared NMPs. Various parameters, such as the effects of the NMP dosage, the pH value of the solution, the initial concentration of $\mathrm{MO}$, and the amount of $\mathrm{H}_{2} \mathrm{O}_{2}$ on the degradation efficiency of $\mathrm{MO}$ were studied. The $\mathrm{MO}$ degradation efficiency could be increased by approximately $100 \%$ by increasing the dosages of the NMPs and $\mathrm{H}_{2} \mathrm{O}_{2}$ to certain limits, after which in both cases the degradation efficiency was reduced when an excess amount was added. The MO degradation efficiency was found to be $100 \%$ at $\mathrm{pH} 2.0$ and 2.5 with the $10 \mathrm{mg} / \mathrm{L}$ of initial concentration of the MO. The degradation of MO by ultrasonically dispersed NMPs was appropriate with the pseudo-first-order kinetics.
\end{abstract}

Keywords: Degradation, Kinetic study, Mechanism, Methyl orange, Nano-metallic particles, Ultrasound

\section{Introduction}

More than 100,000 dyes are available commercially and in use in various industries. Approximately 0.7 million tons of synthetic dyes are generated annually worldwide. However, nearly $15 \%$ of the dye is lost during the dyeing process, becoming released into the environment via industrial effluent-discharge processes [1]. Among all dyes, azo dyes with nitrogen double bonds (-N $=\mathrm{N}$-), characterize the major group of synthetic dyes. These types of dyes have been applied in the cosmetics, textile, paper-making, and printing industries [1-2]. They are a waste material in the printing and dyeing processes and are accordingly discharged in the water system. Hence, the removal of azo dyes has been attracting extensive consideration worldwide [3].

Biological and physical techniques for the removal of azo dyes are not very effective, because of the presence of aromatic rings and the stability of the azo dyes' molecules [4-5]. In addition, wastewater from textile-dyeing facilities is difficult to treat because of its highly fluctuating composition and color variability, depending on the dyestuff, fabric, and concentration of added fixing compounds [6]. However, effective and environmentally friendly techniques are necessary for the proper treatment of azo dyes in wastewater. Fenton-like degradation is a rapid and low-cost technique that is easy to apply on an industrial scale to remove the azo dyes from wastewater [7]. In the Fenton-like process, hydroxyl radicals are generated by oxidation using hydrogen peroxide [8-9]. Currently, nanoparticles are applied to remove azo dyes; these particles include zero-valent iron $\left(\mathrm{Fe}^{0}\right)$ [10-11], zero-valent copper [1], zero-valent zinc [12], and hollow cobalt nanoparticles [2]. Recently, nano-metallic particles (NMPs) have emerged as a promising agent for treating phenol in wastewater [8, 13-14].

Advanced oxidation processes (AOPs) are processes in which hydroxyl radicals are generated in a liquid system and then attack organic pollutants [13]. Ultrasonic cavitation, also considered as an AOP, has also been applied for the degradation of azo dyes $[1,11]$. An application of an ultrasonic process with nano-catalysts in the presence of hydrogen peroxide increases the rate of the oxidative degradation of azo dyes [14]. Extremely reactive hydroxyl radicals are generated in a liquid medium because of cavitational
This is an Open Access article distributed under the terms of the Creative Commons Attribution Non-Commercial License (http://creativecommons.org/licenses/by-nc/3.0/) which permits unrestricted non-commercial use, distribution, and reproduction in any medium, provided the original work is properly cited.
Received November 11, 2016 Accepted February 10, 2017

${ }^{\dagger}$ Corresponding author

Email: jiwansingh95@gmail.com / reddyjchem@gmail.com Tel: : +91-522-2995606 / Tel: 82-2-940-5496

Copyright (C) 2017 Korean Society of Environmental Engineers 
effects [15]. However, no study of the application of NMPs to the degradation of azo dye (methyl orange, MO) has been published earlier.

Therefore, this study was done to evaluate the degradation of MO by NMPs under a heterogeneous Fenton-like process. NMPs were recovered from the fine fraction $(<0.25 \mathrm{~mm})$ of automobile-shredder residue (ASR), which is highly contaminated with heavy metals [16-17]. In many countries, ASR waste is dumped into landfills, increasing the degree of groundwater pollution [16, 18]. In this study, the fine fraction of ASR was used, because it consists of highly hazardous materials because of the availability of high levels of heavy metals [16]. In this study, the degradation efficiency of MO by NMPs is established, and we investigate the effects of the initial MO concentration, the amount of $\mathrm{H}_{2} \mathrm{O}_{2}$, the initial solution $p \mathrm{H}$, the ultrasonic treatment time, and the $\mathrm{NMP}$ dose on the degradation efficiency of the MO removal process.

\section{Materials and Methods}

\subsection{Preparation of NMPs from the Leaching Liquor of ASR}

The details of the leaching conditions are as follows: $1.0 \mathrm{M}$ of $\mathrm{HNO}_{3}$, a liquid-to-solid ratio of $10 \mathrm{~mL} / \mathrm{g}$, and an ultrasound power level of $500 \mathrm{~W}$. To increase heavy-metal extraction from the ASR, an ultrasonic generator (VCX-500, Sonics \& Materials, Inc., USA) was used for $1 \mathrm{~h}$. A leaching solution (100 mL) was used for the synthesis of NMPs, and the $p \mathrm{H}$ of the solution was adjusted to 7.0 with $1.0 \mathrm{M}$ of $\mathrm{NaOH}$. The synthesis of the NMPs was completed according to Singh and Lee [18]. A 0.1 M solution of $\mathrm{NaBH}_{4}$ was added dropwise to a leaching solution at a $\mathrm{pH}$ of 7.0. Subsequently, the mixture was stirred using a temperature-controlled magnetic stirrer at $25 \pm 0.5^{\circ} \mathrm{C}$. The solution mixture became black in color, indicating that the metal ions were converted into NMPs. The separation of the NMPs was done by centrifugation at $4,000 \mathrm{rpm}$ for $30 \mathrm{~min}$. The recovered NMPs were washed several times with distilled water and then cleaned with ethyl alcohol to remove the excess $\mathrm{NaBH}_{4}$. The NMPs were afterward dried in a vacuum at $50^{\circ} \mathrm{C}$. Fig. 1 shows a flowchart of the NMP preparation process and their application to remove $\mathrm{MO}$ from water.

\subsection{MO Degradation under Ultrasonic Irradiation}

The ultrasonic irradiation of the MO solution was carried out in an air atmospheric condition using an ultrasonic generator equipped with a titanium horn transducer. The test of the degradation of MO by NMPs was carried out in $250 \mathrm{~mL}$ glass beaker under ultrasonic power of $500 \mathrm{~W}$ and a frequency of $20 \mathrm{kHz}$. Subsequently, $100 \mathrm{~mL}$ of the MO solution (at various concentrations) was taken in a beaker holding appropriate amounts of NMP particles; in the same mixture proper amounts of $\mathrm{H}_{2} \mathrm{O}_{2}$ were added. The effects of the different parameters, such as initial MO concentration, the amount of $\mathrm{H}_{2} \mathrm{O}_{2}$, the NMP dosage, and the $\mathrm{pH}$ value of the $\mathrm{MO}$ solution. On the degradation of $\mathrm{MO}$ were considered. The $p \mathrm{Hs}$ of the MO solution were adjusted at 2, 2.5, 3, 3.5, and 4 with $0.1 \mathrm{M} \mathrm{HCl}$ and $0.1 \mathrm{~N} \mathrm{NaOH}$. The samples were taken after reaction times of $5,10,15,20,25,30,35$, and $40 \mathrm{~min}$. The residual MO concentrations were then analyzed after the samples were filtered using $0.45 \mu \mathrm{m}$ filter paper. The degradation rate of MO by the NMPs was also measured without the addition of $\mathrm{H}_{2} \mathrm{O}_{2}$ under ultrasonic irradiation. A calibration curve

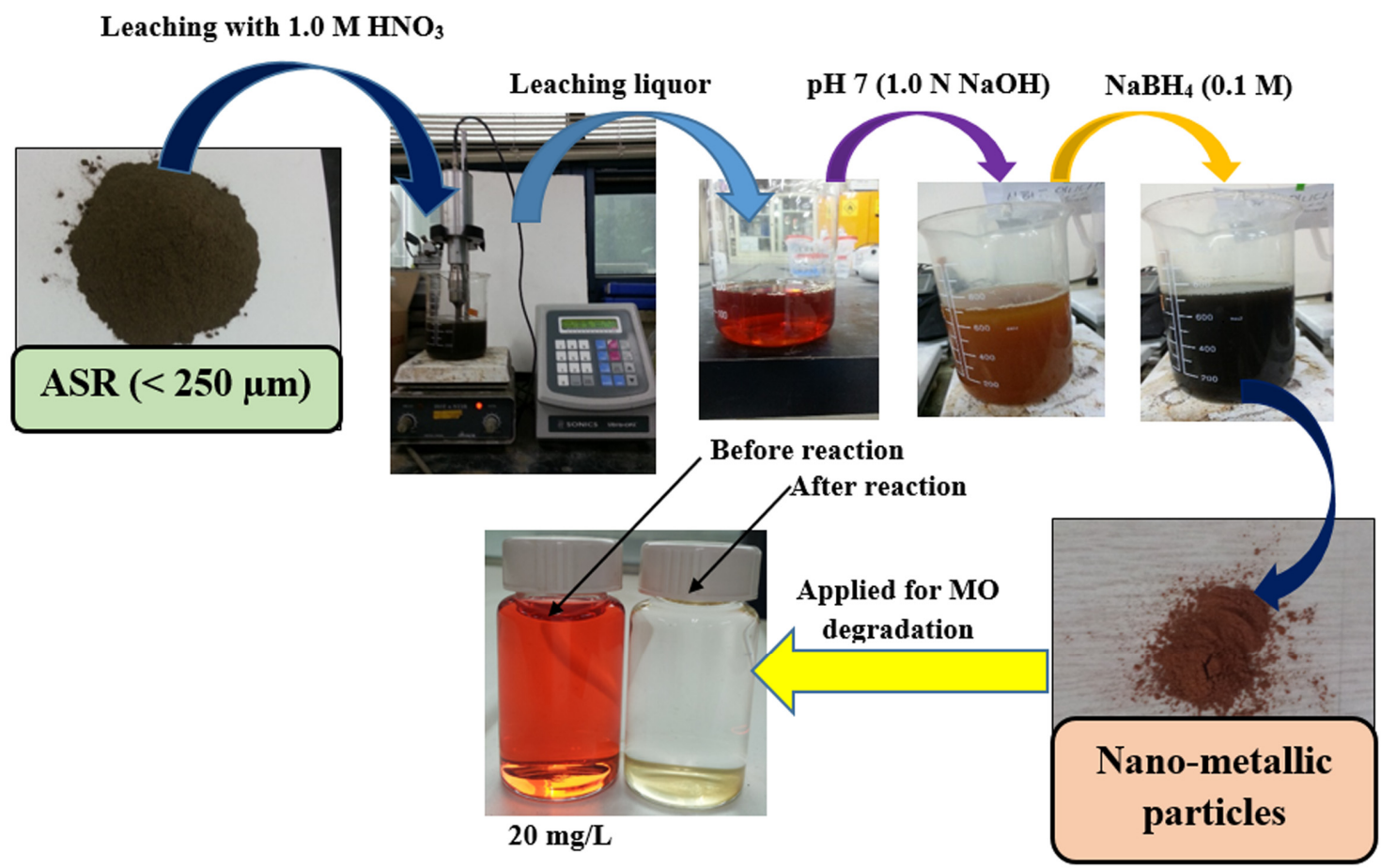

Fig. 1. Flow chart of the NMP preparation process and its application for MO removal. 
was obtained using the standard MO solution with different known concentrations. All experiments were carried out at least three times.

The dye degradation efficiency of MO was calculated using Eq. (1):

$$
\text { MO degradation efficiency }=\left(\left(C_{0}-C_{t}\right) / C_{0}\right) \times 100
$$

where $C_{0}$ and $C_{t}$ denote the concentrations of $\mathrm{MO}$ at time 0 and $t$, respectively.

\subsection{Analysis}

The concentrations of elements in the leaching liquor of ASR were measured by means of inductively coupled plasma atomic emission spectroscopy (ICP-AES) (Perkin Elmer Inc., Optima 2000 DV). A UV-visible spectrophotometer (UV 1601, Shimadzu) was used to measure the residual MO concentration at a maximum wavelength $\left(\lambda_{\max }\right)$ of $465 \mathrm{~nm}$. A pH meter (Orion 3 Star, Thermo Scientific) was used to measure the solution $p H$. The NMPs were analyzed by a scanning electron microscope (SEM; S-4300CX; Hitachi, Japan). X-ray photoelectron spectroscopy (XPS) and Fourier-transform infrared spectrophotometry (FT-IR) analyses of the NMPs before and after the reaction were carried out using an Escalab-210 electron spectrophotometer (Spain) and an ISF 66/S spectrophotometer (Bruker), respectively. The XPS system was used to define the elemental states of various metals, and FT-IR was applied to describe the functional groups available on the surfaces of the NMPs.

\subsection{Kinetics of MO Degradation}

The degradation of MO by NMPs under ultrasonic irradiation was demonstrated by a pseudo-first-order reaction [13, 19],

$$
\ln \left(C_{t} / C_{0}\right)=-k t
$$

where $C_{0}$ signifies the initial concentration of $\mathrm{MO}(\mathrm{mg} / \mathrm{L})$ and $C_{t}$ is the concentration of $\mathrm{MO}$ at time $t$ (min), with $k$ representing the pseudo-first-order constant $\left(\mathrm{min}^{-1}\right)$.

The degradation of the phenol by NMPs was also analyzed by pseudo-second-order reaction kinetics [20],

$$
1 / C_{t}=\left(1 / C_{0}\right)+k_{2} t
$$

The slope of the plot between $1 / C_{t}$ and time yields the pseudo-second-order rate constant $k$.

\section{Results and Discussion}

\subsection{Characterization of the NMPs}

The ICP analysis indicated that the concentrations of Fe, Mn, $\mathrm{Ni}, \mathrm{Cu}, \mathrm{Zn}, \mathrm{Cr}$, and $\mathrm{Pb}$ in the ASR leaching liquor were 639.0, 35.0, 3.9, 41.5, 281.2, 2.5, and $41.2 \mathrm{mg} / \mathrm{L}$, respectively. An SEM analysis of NMPs is given elsewhere [13]. The SEM image of the nanoparticles was found to be spherical and structurally rough on the surface, signifying that abundant reactive sites are available on the surfaces of the NMPs. The spherical NMPs forming an aggregate structure may result from magnetic interactions between the nanoparticles [8].

Fig. 2 shows the FT-IR spectra of the NMPs as measured before and after the reaction. Band peaks at 980.0 and 1,391.9 $\mathrm{cm}^{-1}$ were observed on the surfaces of the NMPs; these are confirmation of $\mathrm{M}-\mathrm{O}$ stretching connected to metal oxides (such as $\mathrm{MO}, \mathrm{M}_{2} \mathrm{O}_{3}$, and $\mathrm{M}_{3} \mathrm{O}_{4}$.), characteristic of the accessibility of metal oxides on the surfaces of the NMPs $[13,21]$. The FT-IR spectra of the NMPs showed broad bands at $3,308.0 \mathrm{~cm}^{-1}$, which could be assigned to the hydroxyl group $(\mathrm{O}-\mathrm{H})$. After the reaction, the intensity of this band was reduced and the peak was found to be broader. After the reaction, the peaks of the bands were shifted from 1,638.6 to $1,608.4 \mathrm{~cm}^{-1}$, from $1,391.9$ to $1,413.8 \mathrm{~cm}^{-1}$, and from 980.0 to $1,024.5 \mathrm{~cm}^{-1}$, likely corresponding to $\mathrm{M}-\mathrm{O}$ stretching and representing oxidized form of NMPs in the reaction [13].

XPS was further applied to study the surface chemical compositions of NMPs. As shown in Fig. 3(a), the XPS analyses showed that $\mathrm{C}, \mathrm{O}$, and few metals ( $\mathrm{Fe}, \mathrm{Cu}, \mathrm{Zn}$, and $\mathrm{Mn}$ ) existed on the surfaces of the NMPs. The presence of $\mathrm{O}$ likely stems from the fact that the NMPs were easily oxidized partially by oxygen when exposed to air. Two C (1s) peaks were identified at 284.5 and $287.6 \mathrm{eV}$, allotted to C-C/C-H and COO bonds, respectively [22-23]. However, after the reaction, these peaks were shifted to 283.4 and $286.99 \mathrm{eV}$, respectively. The $\mathrm{O}$ (1s) peak at $528.4 \mathrm{eV}$ was recognized as showing the availability of oxides on the surfaces of the NMPs, whereas after the reaction, the intensity of this peak was reduced, possibly due to the conversion of oxides into metallic ions [14]. Metals existed on the surfaces of the NMPs in the form of elements, oxides of metals, and carbonates of metals. The peak of the binding energy (BE) of $\mathrm{Cu}$ was observed at $932.5 \mathrm{eV}$, representative of $\mathrm{Cu}^{0}$ [24]; moreover, this peak did not appear after the reaction with MO (Fig. 4(a)), indicating that $\mathrm{Cu}^{0}$ was oxidized to $\mathrm{Cu}^{+} / \mathrm{Cu}^{2+}$ [25]. The XPS spectra of Fe (2p) in Fig. 4(b) show two peaks, with BE values of 711.1 and $724.5 \mathrm{eV}$.

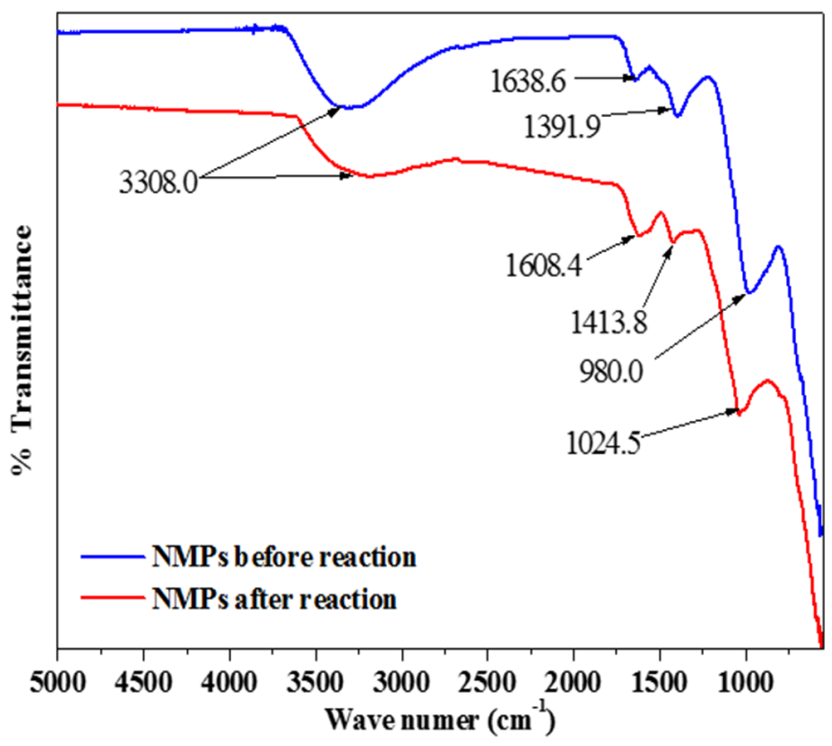

Fig. 2. FT-IR spectra of NMPs before and after the reaction. 

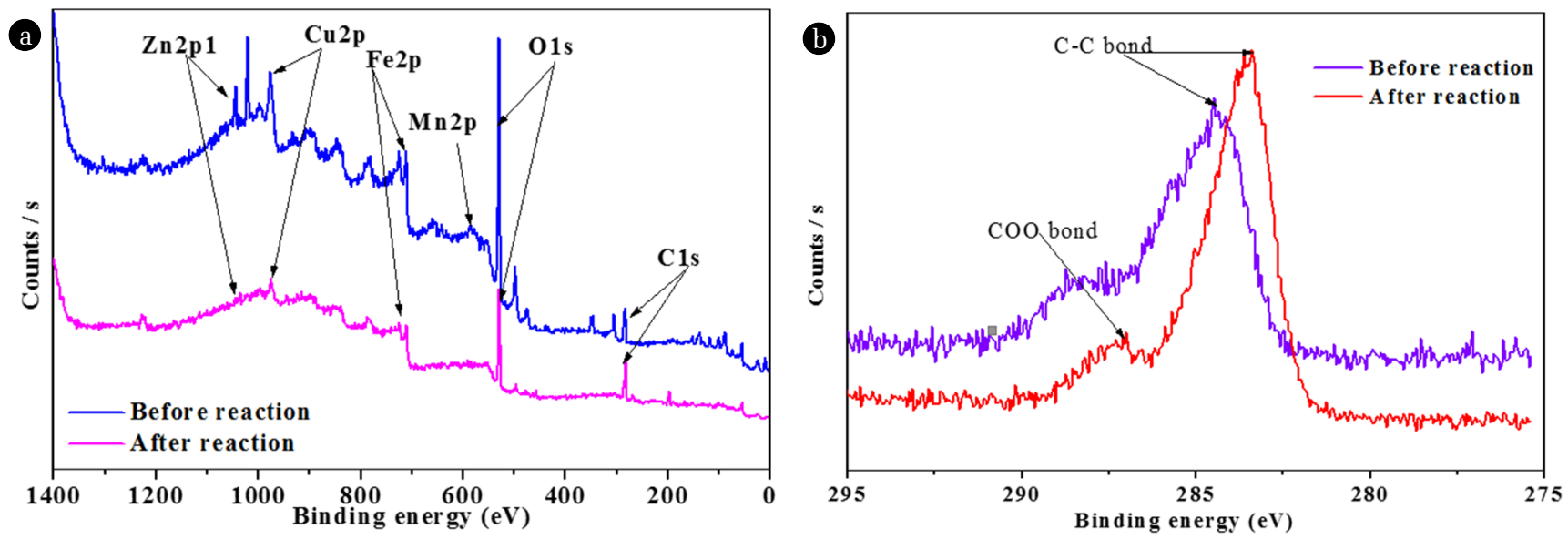

Fig. 3. XPS analysis of the NMPs before and after the reaction: (a) survey analysis, (b) C 1s.
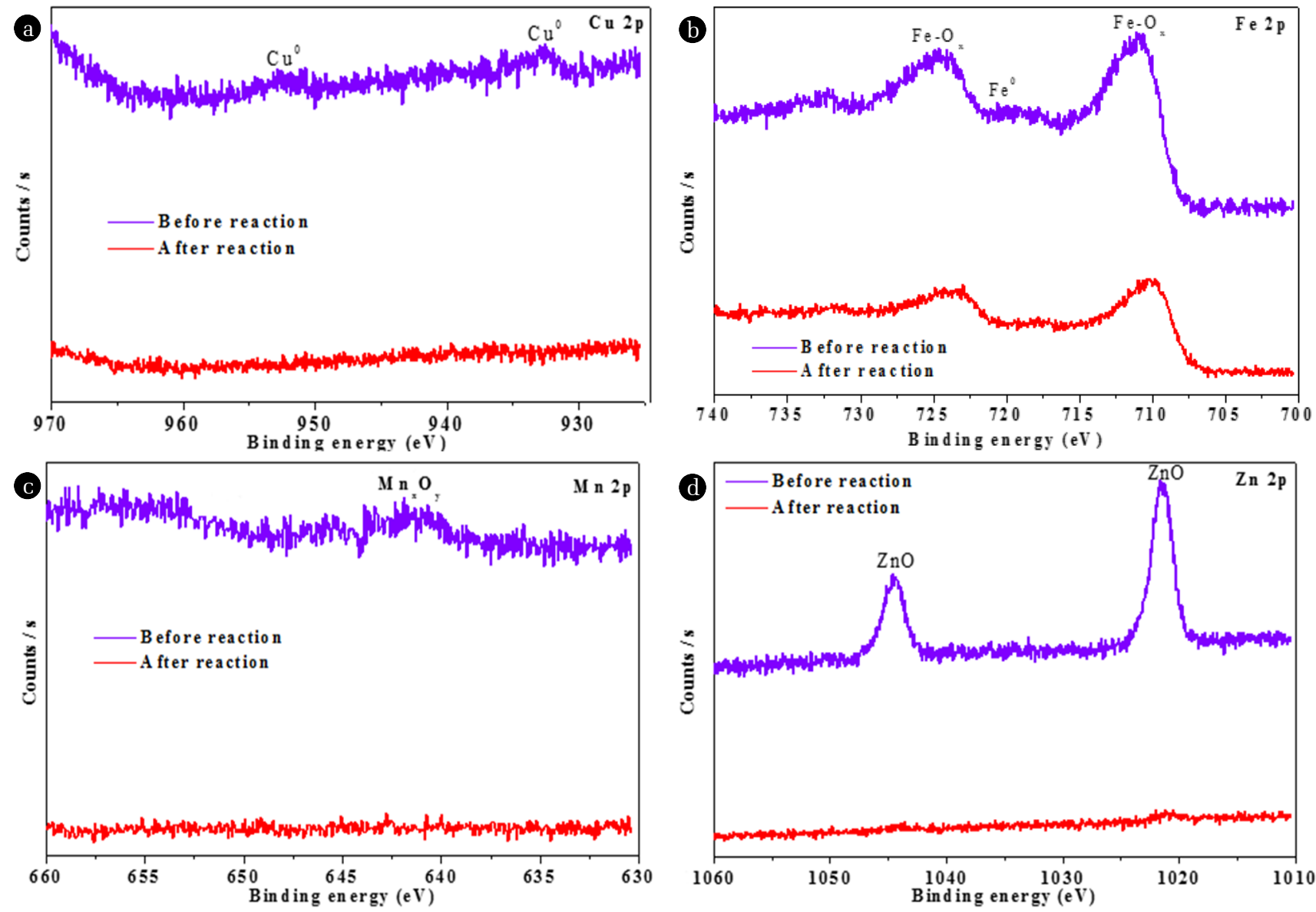

Fig. 4. XPS analysis of the NMPs before and after the reaction: (a)

Cu 2p, (b) Fe 2p, (c) Mn 2p, and (d) Zn 2p.

These broad peaks could be assigned to iron oxides $\left(\mathrm{Fe}_{2} \mathrm{O}_{3}\right)$, indicating that the surfaces of the NMPs were covered with an iron-oxide film which likely formed during the synthesis process [1, 8]. The intensities of both peaks decreased after the reaction, confirming that oxides of $\mathrm{Fe}$ were involved in the reaction. The $\mathrm{BE}$ of the $\mathrm{Mn} 2 \mathrm{p}$ before the reaction showed a peak at $641.9 \mathrm{eV}$, assigned to $\mathrm{MnO}$ and $\mathrm{MnO}_{2}$ [26]. However, after the reaction, this peak was not seen, signifying that the oxides of Mn contributed to the degradation of MO (Fig. 4(c)). The XPS spectra of Zn (2p) in Fig. 4(d) shows two peaks, at BEs of 1021.6 and $1044.6 \mathrm{eV}$, 
which were assigned to $\mathrm{ZnO}$ [27]. These peaks were not seen after the reaction, confirming the participation of $\mathrm{ZnO}$ in the degradation of MO.

\subsection{Removal of MO by NMPs}

\subsubsection{Effect of the dosages of NMPs}

Fig. 5 displays the effect of different NMP dosages $(0.025,0.05$, 0.10 , and $0.150 .20 \mathrm{~g} / \mathrm{L}$ ) on the degradation of MO (experimental conditions: $p \mathrm{H}$ of 3.0, the initial MO concentration of $20 \mathrm{mg} / \mathrm{L}$, $\mathrm{H}_{2} \mathrm{O}_{2}$ concentration of $50 \mathrm{mM}$, a reaction time of $40 \mathrm{~min}$, and ultrasound power of $500 \mathrm{~W}$ ). As shown in Fig. 6(a), the degradation of MO was found to be $99.9 \%$ with a dose of $0.050 \mathrm{mg} / \mathrm{L}$ at a reaction time of $40 \mathrm{~min}$, which can be explained as that, when

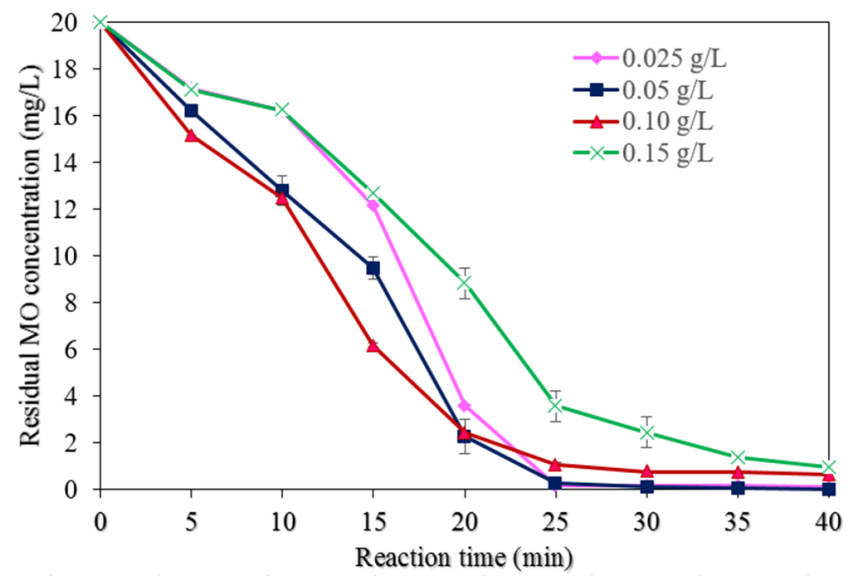

Fig. 5. Effects of different dosages of NMPs on the MO degradation process (experimental conditions: $\mathrm{MO}$ concentration, $20 \mathrm{mg} / \mathrm{L}$; pH 3.0; concentration of $\mathrm{H}_{2} \mathrm{O}, 50 \mathrm{mM}$; ultrasound power. $500 \mathrm{~W}$ ). the dose of NMPs increased, then the number of reaction sites also increased [8]. However, upon a further increase of the dose, the MO degradation efficiency was reduced to $95.1 \%$, most likely because of the scavenging effect of hydroxyl radicals and the agglomeration of NMPs in the reaction system [28].

The values of the pseudo-first-order reaction rate constant $(k)$ and correlation coefficient $\left(R^{2}\right)$ for the MO degradation rate with different dosages of NMPs were found from the plot of $\ln \left(\mathrm{C} / \mathrm{C}_{0}\right)$ vs. the reaction time (Fig. 7(a)). Table 1 shows the values of $k_{1}$ and $R^{2}$ for diverse dosages of NMPs. When the quantity of NMPs was increased up to $0.050 \mathrm{~g} / \mathrm{L}$, the values of $k_{1}$ and $k_{2}$ were increased. The value of $k_{1}$ was reduced approximately $47.8 \%$; however, the value of $k_{2}$ was reduced to $89.3 \%$ with a further increase in the dosage of NMPs.

\subsubsection{Effect of the solution $\mathrm{pH}$ on $\mathrm{MO}$ degradation by NMPs}

Fig. 8 displays the effects of the $p \mathrm{H}$ values on the $\mathrm{MO}$ degradation process by NMPs (experimental conditions: MO concentration of $20 \mathrm{mg} / \mathrm{L}$, NMP dose of $0.05 \mathrm{~g} / \mathrm{L}$, concentration of $\mathrm{H}_{2} \mathrm{O}_{2}$ of $50 \mathrm{mM}$, reaction time of $40 \mathrm{~min}$, and an ultrasound power level of $500 \mathrm{~W}$ ). The highest and most rapid MO degradation rate was achieved at $p \mathrm{H}$ levels of 2.0 and 2.5. The degradation efficiency of MO was found to be approximately $100 \%$ at reaction times of 25 and $30 \mathrm{~min}$ at $\mathrm{pH}$ levels of 2.0 and 2.5, respectively (Fig. 6(b)).

However, the lowest MO degradation efficiency was found to be approximately $25 \%$ at a $p \mathrm{H}$ of 4.0 at a reaction time of $40 \mathrm{~min}$. The $\mathrm{MO}$ degradation rate decreased with an increase in the $p \mathrm{H}$ from 2.0 to 4.0. This arose due to the decay of $\mathrm{H}_{2} \mathrm{O}_{2}$ with the decrease in catalytic activity of the NMPs [29]. The values of $k_{1}$ and $R^{2}$ for the MO degradation rate with different $p \mathrm{H}$ values were found from the plot of $\ln \left(\mathrm{C} / \mathrm{C}_{0}\right)$ vs. the reaction time (Fig. 7(b)). Table 1 shows the values of $k_{1}$ and $R^{2}$ with

Table 1. A Kinetic Results for MO Degradation by Ultrasonically Dispersed NMPs under Fenton-like Process

\begin{tabular}{|c|c|c|c|c|c|c|c|}
\hline \multirow{2}{*}{$\begin{array}{c}\text { NMPs dose } \\
(\mathrm{g} / \mathrm{L})\end{array}$} & \multirow{2}{*}{$\begin{array}{c}\mathrm{C}_{0}(\mathrm{MO}) \\
(\mathrm{mg} / \mathrm{L})\end{array}$} & \multirow{2}{*}{$\begin{array}{c}\text { Initial } \\
\mathbf{p H}\end{array}$} & \multirow{2}{*}{$\begin{array}{c}\mathrm{H}_{2} \mathrm{O}_{2} \text { conc. } \\
(\mathrm{mM})\end{array}$} & \multicolumn{2}{|c|}{ Pseudo-first-order } & \multicolumn{2}{|c|}{ Pseudo-second-order } \\
\hline & & & & $k_{1}\left(\min ^{-1}\right)$ & $\mathbf{R}^{2}$ & $k_{2}(\mathrm{~L} / \mathrm{mg} \min )$ & $\mathbf{R}^{2}$ \\
\hline 0.025 & 20 & 3.0 & 50 & 0.157 & 0.8698 & 0.215 & 0.8309 \\
\hline 0.05 & 20 & 3.0 & 50 & 0.194 & 0.9409 & 0.610 & 0.7027 \\
\hline 0.10 & 20 & 3.0 & 50 & 0.101 & 0.9439 & 0.044 & 0.9128 \\
\hline 0.15 & 20 & 3.0 & 50 & 0.082 & 0.9429 & 0.023 & 0.7869 \\
\hline 0.05 & 10 & 3.0 & 50 & 0.166 & 0.9548 & 1.660 & 0.6257 \\
\hline 0.05 & 40 & 3.0 & 50 & 0.131 & 0.9177 & 0.556 & 0.6437 \\
\hline 0.05 & 80 & 3.0 & 50 & 0.128 & 0.9146 & 0.004 & 0.8449 \\
\hline 0.05 & 20 & 2.0 & 50 & 0.302 & 0.9438 & 0.001 & 0.9756 \\
\hline 0.05 & 20 & 2.5 & 50 & 0.209 & 0.9565 & 0.834 & 0.5734 \\
\hline 0.05 & 20 & 3.5 & 50 & 0.042 & 0.9547 & 0.053 & 0.7005 \\
\hline 0.05 & 20 & 4.0 & 50 & 0.008 & 0.9830 & 0.022 & 0.8348 \\
\hline 0.05 & 20 & 3.0 & 0 & 0.005 & 0.9656 & 0.003 & 0.9705 \\
\hline 0.05 & 20 & 3.0 & 25 & 0.113 & 0.9222 & 0.056 & 0.8556 \\
\hline 0.05 & 20 & 3.0 & 100 & 0.124 & 0.9466 & 0.092 & 0.6995 \\
\hline 0.05 & 20 & 3.0 & 150 & 0.118 & 0.9461 & 0.071 & 0.7890 \\
\hline
\end{tabular}



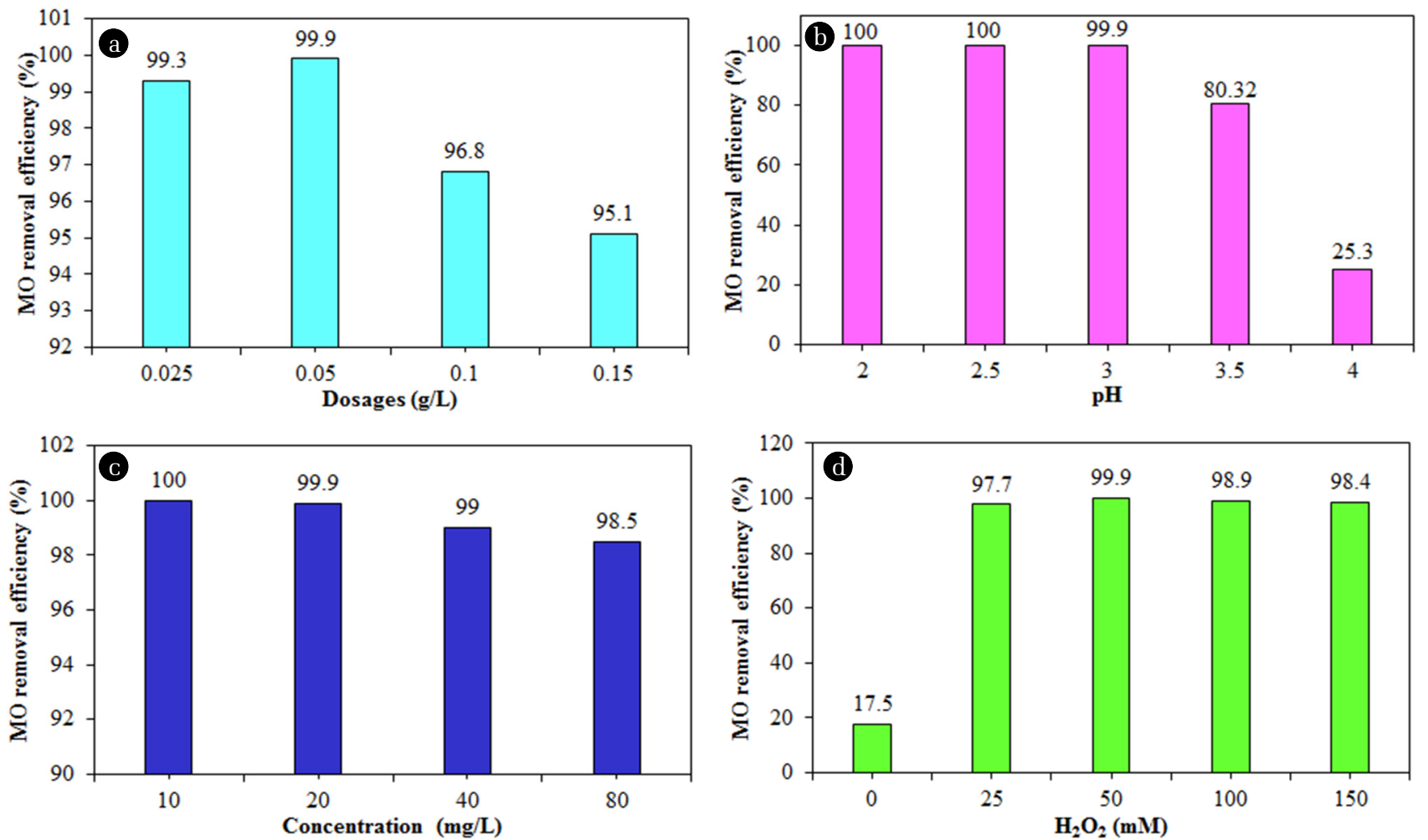

Fig. 6. Changes in MO degradation efficiency rates with different (a) NMP dosages, (b) $p H$ levels, (c) MO concentrations, and (d) $\mathrm{H}_{2} \mathrm{O}_{2}$ concentrations.
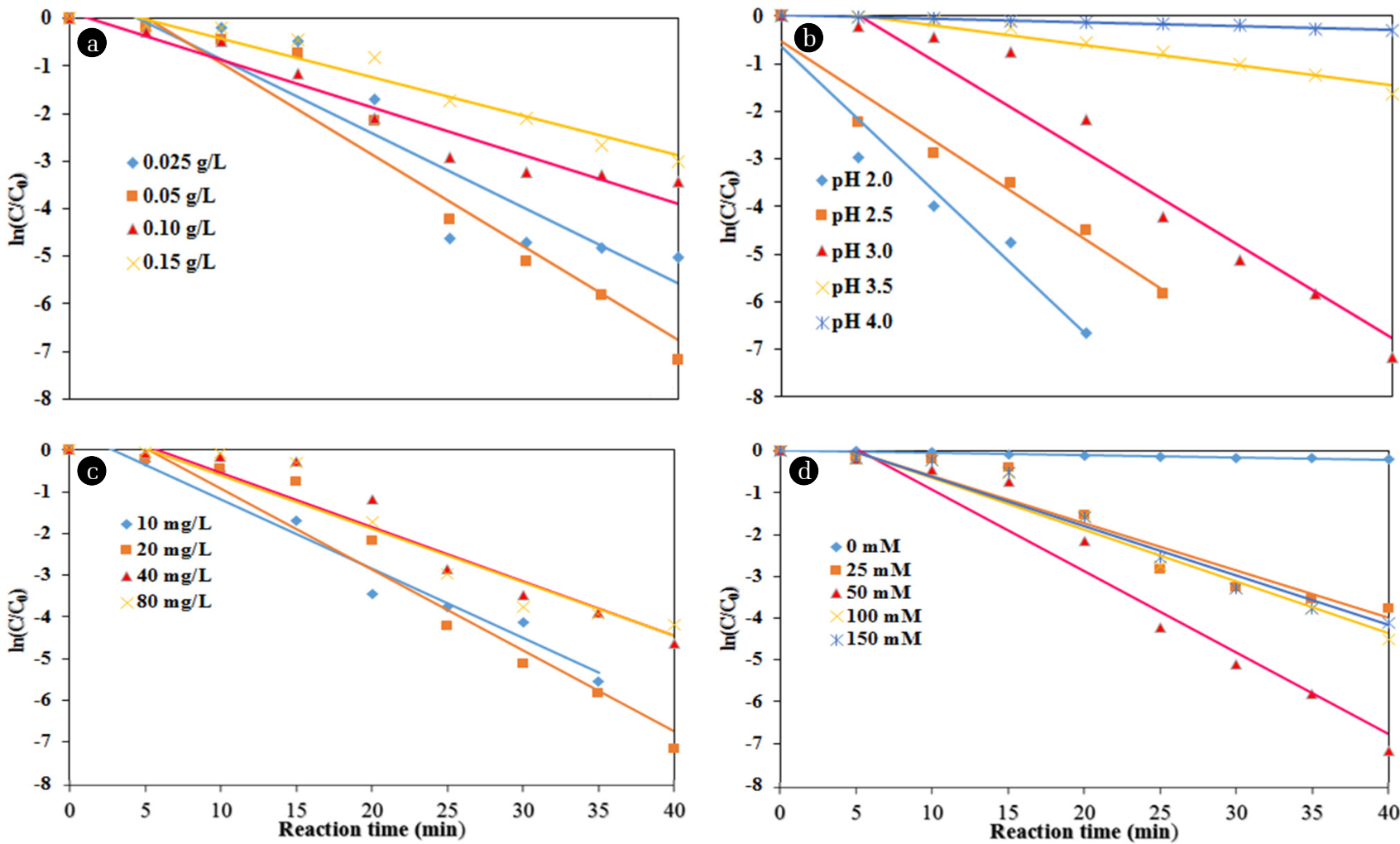

Fig. 7. Kinetic study of MO degradation by NMPs with different parameters: (a) NMP dosages, (b) $p H$ values, (c) initial concentrations of MO, and (d) amounts of $\mathrm{H}_{2} \mathrm{O}_{2}$. 


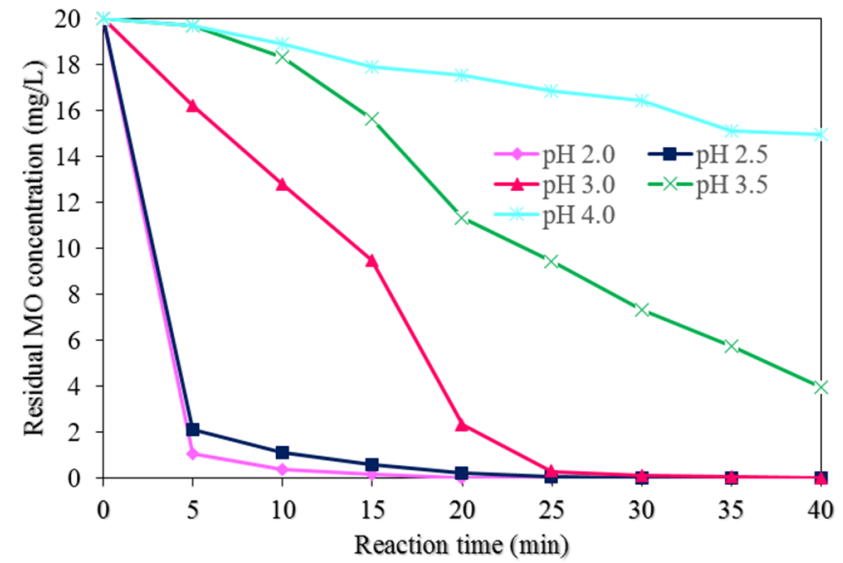

Fig. 8. Effects of different values of $\mathrm{pH}$ on the $\mathrm{MO}$ degradation (test condition: MO concentration. $20 \mathrm{mg} / \mathrm{L}$; NMP dose, $0.05 \mathrm{~g} / \mathrm{L}$; concentration of $\mathrm{H}_{2} \mathrm{O}_{2}, 50 \mathrm{mM}$; and ultrasound power, $500 \mathrm{~W}$ ).

different $p \mathrm{H}$ values. The value of $k_{1}$ was reduced about $97 \%$ when the value of $p \mathrm{H}$ was increased from 2.0 to 4.0. However, the value of $k_{2}$ was increased with the increase in the $p \mathrm{H}$ of the solution.

\subsubsection{Effect of the initial MO concentration on $M O$ degradation} Fig. 9 exhibits the effect of different initial MO concentrations on the degradation of MO by NMPs (experimental conditions: a NMP dose of $0.05 \mathrm{~g} / \mathrm{L}$, a $p \mathrm{H}$ of 3.0 , a $\mathrm{H}_{2} \mathrm{O}_{2}$ concentration of $50 \mathrm{mM}$, a reaction time of $40 \mathrm{~min}$, and ultrasound power of 500 $\mathrm{W})$. The degradation efficiency of MO was reduced from 100 to $98.5 \%$ with an increase in the MO concentration from 10 to $80 \mathrm{mg} / \mathrm{L}$ at a reaction time of $40 \mathrm{~min}$ (Fig. 6(c)). This result indicates rapid occupying of all the active sites of the NMPs by the MO molecules at a high level of MO, resulting in a decrease in the production of hydroxyl radicals in the sono-catalysis process [13, 27]. The residual concentrations of $\mathrm{MO}$ achieved were found to be $0,0.016,0.39$, and $1.21 \mathrm{mg} / \mathrm{L}$ at a reaction time of $40 \mathrm{~min}$ with initial MO concentrations of $10,20,40$, and $80 \mathrm{mg} / \mathrm{L}$,

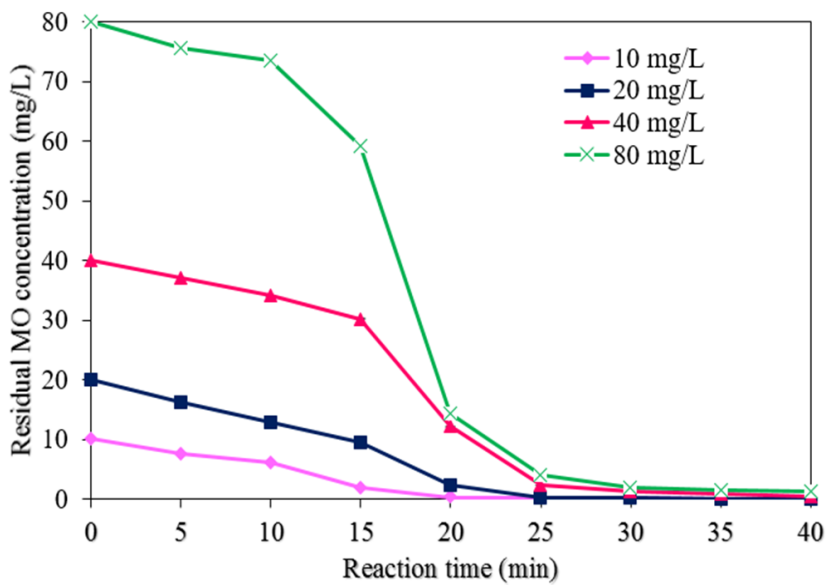

Fig. 9. Effects of different initial concentrations of $M O$ on the $M O$ degradation (test conditions: $\mathrm{pH}, 3.0 ; \mathrm{NMP}$ dosage, $0.05 \mathrm{~g} / \mathrm{L}$; concentration of $\mathrm{H}_{2} \mathrm{O}_{2}, 50 \mathrm{mM}$; ultrasound power, $500 \mathrm{~W}$ ). respectively. The values of $k_{1}$ and $R^{2}$ for the MO degradation rate with different concentrations of $\mathrm{MO}$ were found from the plot of $\ln \left(\mathrm{C} / \mathrm{C}_{0}\right)$ vs. the reaction time (Fig. 7(c)). Table 1 shows the values of $k_{1}, k_{2}$, and $R^{2}$ with different concentrations of MO. When the MO concentration was increased from 10 to $80 \mathrm{mg} / \mathrm{L}$, the values of $k_{1}$ and $k_{2}$ were decreased to $97.4 \%$ and $99.8 \%$, respectively.

\subsubsection{Effect of the amount of $\mathrm{H}_{2} \mathrm{O}_{2}$}

Fig. 10 demonstrates the effects of different concentrations of $\mathrm{H}_{2} \mathrm{O}_{2}$ on the MO degradation rate (test conditions: a NMP dosage of $0.05 \mathrm{~g} / \mathrm{L}$, a $p \mathrm{H}$ of 3.0 , an initial $\mathrm{MO}$ concentration of $20 \mathrm{mg} / \mathrm{L}$, a reaction time of $40 \mathrm{~min}$, and ultrasound power of $500 \mathrm{~W}$ ). The degradation of MO by NMPs was very slow in the absence of $\mathrm{H}_{2} \mathrm{O}_{2}$, because there was insufficient production of hydroxyl radicals in the system. As displayed in Fig. 6(d), the degradation efficiency of MO increased considerably, from approximately $17 \%$ to $100 \%$ by increasing $\mathrm{H}_{2} \mathrm{O}_{2}$ concentration from $0 \mathrm{mM}$ to $50 \mathrm{mM}$, perhaps because of enough hydroxyl radicals were produces [27]. MO degradation was achieved very rapidly with $50 \mathrm{mM}$ of $\mathrm{H}_{2} \mathrm{O}_{2}$. The degradation of $\mathrm{MO}$ was increased by an increasing concentration of $\mathrm{H}_{2} \mathrm{O}_{2}$, to $50 \mathrm{mM}$. However, upon a further increase in the amount of $\mathrm{H}_{2} \mathrm{O}_{2}$, the degradation efficiency of $\mathrm{MO}$ was reduced slightly. The MO degradation may be possible with $\mathrm{H}_{2} \mathrm{O}_{2}$ with ultrasound (without NMPs); however, its degradation with only $\mathrm{H}_{2} \mathrm{O}_{2}$ is somewhat difficult, because there is no external agent to break down the $\mathrm{H}_{2} \mathrm{O}_{2}$. The values of $k$ and $R^{2}$ for the MO degradation rate with different concentrations of $\mathrm{H}_{2} \mathrm{O}_{2}$ were obtained from the plot of $\ln \left(\mathrm{C} / \mathrm{C}_{0}\right)$ vs. the reaction time (Fig. $7(\mathrm{~d})$ ). Table 1 shows the values of $k_{1}$, $k_{2}$ and $R^{2}$ with different amounts of $\mathrm{H}_{2} \mathrm{O}_{2}$. The values of $k_{1}$ and $k_{2}$ were increased with an increase in the amount of $\mathrm{H}_{2} \mathrm{O}_{2}$, from $0 \mathrm{mM}$ to $100 \mathrm{mM}$; however, these values were decreased with a further addition of $\mathrm{H}_{2} \mathrm{O}_{2}$.

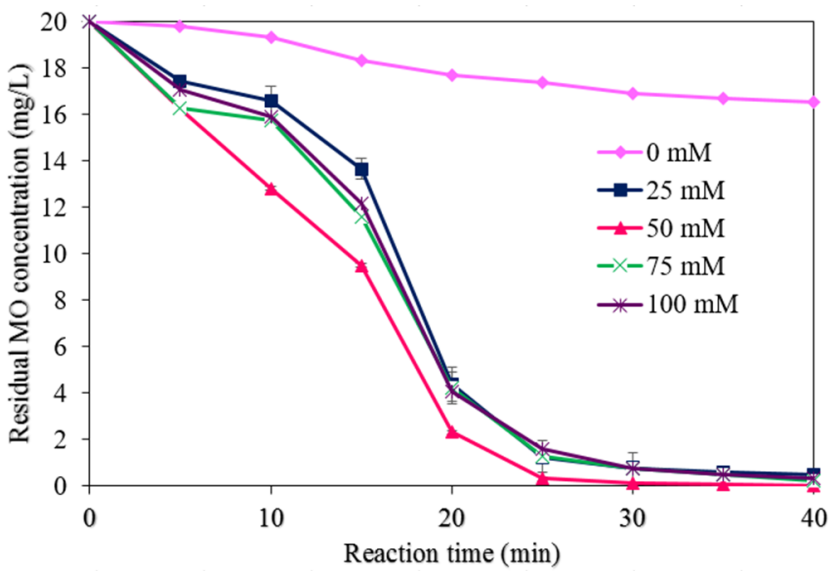

Fig. 10. Effects of different amounts of $\mathrm{H}_{2} \mathrm{O}_{2}$ on the $\mathrm{MO}$ degradation process (test conditions: $\mathrm{MO}$ concentration, $20 \mathrm{mg} / \mathrm{L} ; \mathrm{pH}, 3.0$; NMP dose, $0.05 \mathrm{~g} / \mathrm{L}$; ultrasound power, $500 \mathrm{~W}$ ).

\subsubsection{Synergistic effects of combined sonication with Fenton's reagent}

The degradation efficiency of MO was found to be approximately 
$100 \%$ for NMPs combined with ultrasonic irradiation and $\mathrm{H}_{2} \mathrm{O}_{2}$. The degradation efficiency of MO by NMPs with a combination of $\mathrm{H}_{2} \mathrm{O}_{2}$ and ultrasound represents the great potential of MO degradation in a very short period of time as compared to only the $\mathrm{NMP} /$ ultrasound power or $\mathrm{H}_{2} \mathrm{O}_{2}$ /ultrasound power combinations. The oxidation of MO by ultrasonically dispersed NMPs in the absence of $\mathrm{H}_{2} \mathrm{O}_{2}$ required more sonication time for the complete degradation of MO. In the ultrasound/NMP system, the MO degradation efficiency was low (16.5\%) at a reaction time of $40 \mathrm{~min}$. However, in the NMP/ultrasound/ $\mathrm{H}_{2} \mathrm{O}_{2}$ system, a $\mathrm{MO}$ degradation efficiency rate of $100 \%$ was achieved. The removal of $\mathrm{MO}$ was low because too few hydroxyl radicals were produced during the sono-advanced Fenton process (AFP). The ultrasonic irradiation effect was more noticeable at room temperature, because most of the bubbles were filled with gas. The increase in the reaction temperature reduced the degradation efficiency, perhaps because the cavitation threshold was lowered. Therefore, more water vapor was produced by the cavitation bubbles; this water vapor could decrease the ultrasonic energy produced during cavitation [30].

\subsection{Possible Mechanism for the Degradation of MO by NMPs}

During the ultrasonic irradiation, both physical and chemical processes, such as configuration, expansion, and adiabatically implosive breakdown of microbubbles in the aqueous system, are involved. These processes could be triggered by the propagation of the ultrasonic wave through acoustic cavitation. The quick breakdown of cavitation bubbles may be coupled with adiabatic heating of the vapor phase of the bubble, which gains great local transitory temperatures and pressures [31]. Under these exciting conditions, water molecules break down through heat energy to generate the extremely reactive radicals ${ }^{\circ} \mathrm{H}$ and ${ }^{\circ} \mathrm{OH}$, and yields $\mathrm{HO}_{2}{ }^{\mathrm{O}}$ in the occurrence of $\mathrm{O}_{2}$ in the aqueous system. Therefore, organic compounds such as MO in close proximity to the bubble/water interface can be exposed to thermal decomposition and/or secondary reactions between the reactive radicals and $\mathrm{MO}$, resulting in their degradation or mineralization [32]. The oxidation of MO by hydroxyl radicals in the sono-AFP is highly probable. Many hydroxyl radicals are generated in an ultrasound/NMP $/ \mathrm{H}_{2} \mathrm{O}_{2}$ system [8, 13, 33]. In the sono-AFP, a pressure pulse is created during the breakdown of a solo cavity, leading to a high rate of production of hydroxyl radicals [15]. The ultrasound irradiation effects during the MO degradation process led to the production of many hydroxyl radicals, which mainly target MO molecules in an aqueous system. Ultrasonic irradiation improves the corrosion of NMPs and successively releases many metal ions. Appropriate amounts of metals cations $\left(\mathrm{M}^{\mathrm{n}+}\right)$ and $\mathrm{H}_{2} \mathrm{O}_{2}$ are required to stimulate the AFP. In the presence of $\mathrm{H}_{2} \mathrm{O}_{2}$, the ultrasound irradiation produces many hydroxyl radicals in an aqueous system (Eq. (4)). The NMPs can react with the added $\mathrm{H}_{2} \mathrm{O}_{2}$, causing the generation of further hydroxyl radicals [8, 33]. The highest rate of $\mathrm{MO}$ degradation was achieved at a $\mathrm{pH}$ of 3.0, which can be attributed the presence of several metal oxides on the surfaces of NMPs. These metal oxides were released shortly at an acidic $p \mathrm{H}$, after which many active sites existed for reaction with MO.

$$
\begin{aligned}
& \mathrm{H}_{2} \mathrm{O}_{2} \longrightarrow 2 \mathrm{JH}^{\mathrm{o}} \\
& \mathrm{H}_{2} \mathrm{O}_{2}+2 \mathrm{OH}^{\mathrm{o}} \rightarrow \mathrm{H}_{2} \mathrm{O}+\mathrm{HO}_{2}{ }^{\mathrm{o}} \\
& \mathrm{O}_{2} \longrightarrow 2 \mathrm{~J}^{\circ} \\
& 2 \mathrm{O}^{\circ}+\mathrm{H}_{2} \mathrm{O} \rightarrow 2 \mathrm{OH}^{\mathrm{o}} \\
& \mathrm{OH}^{\mathrm{o}}+\mathrm{OH}^{\mathrm{o}} \rightarrow \mathrm{H}_{2} \mathrm{O}_{2} \\
& \mathrm{M}^{0} \stackrel{(J)) J)}{\longrightarrow} \mathrm{M}^{\mathrm{n}+}+2 \mathrm{e}^{-} \\
& \mathrm{M}^{0}+n \mathrm{H}^{+} \rightarrow \mathrm{M}^{n+}+n \mathrm{H}_{2} \\
& \mathrm{M}^{n+}+\mathrm{H}_{2} \mathrm{O}_{2} \rightarrow \mathrm{M}^{(n+1)+}+\mathrm{OH}^{\circ}+\mathrm{OH} \\
& \mathrm{NMPs}+n \mathrm{H}^{+}+\mathrm{H}_{2} \mathrm{O}_{2} \rightarrow \mathrm{M}^{n+}+\mathrm{OH}^{\cdot}+\mathrm{OH}^{-} \\
& \mathrm{M}_{\mathrm{x}} \mathrm{O}_{\mathrm{y}}+n \mathrm{H}^{+} \rightarrow n \mathrm{M}^{n+}+n \mathrm{H}_{2} \mathrm{O} \\
& \mathrm{OH}^{\mathrm{o}}+\mathrm{MO} \rightarrow \text { Intermediate }+\mathrm{CO}_{2}+\mathrm{H}_{2} \mathrm{O}
\end{aligned}
$$

Here, $M$ is the metal, $n$ is the number of valences, $O$ denotes the oxides, 'J) J)\}' represents the ultrasound waves, and $x$ and $y$ correspondingly represent the number of moles.

\section{Conclusions}

The degradation of MO by ultrasonically dispersed NMPs was examined with and without the addition of hydrogen peroxide. The removal of MO was found to be affected by the $p \mathrm{H}$ value of the solution and by the NMP and hydrogen peroxide dosages. This study particularly demonstrated that NMPs are highly efficient for MO degradation in the presence of $\mathrm{H}_{2} \mathrm{O}_{2}$ and ultrasonic power. A decrease in the $p \mathrm{H}$ value and the initial concentration of MO increased the degradation efficiency of MO; however, an increase in the NMP and $\mathrm{H}_{2} \mathrm{O}_{2}$ dosages increased the MO degradation efficiency level only up to a certain limit. Excess amounts of NMPs and $\mathrm{H}_{2} \mathrm{O}_{2}$ decreased the MO degradation efficiency. The optimum conditions for the degradation of MO were a NMP dosage of $0.05 \mathrm{~g} / \mathrm{L}$, an $\mathrm{H}_{2} \mathrm{O}_{2}$ concentration of $50 \mathrm{mM}$, an $\mathrm{MO}$ concentration of $40 \mathrm{mg} / \mathrm{L}$, a $p \mathrm{H}$ of 3.0 , ultrasonic power of $500 \mathrm{~W}$, and a reaction time of $40 \mathrm{~min}$. The pseudo-first-order kinetics were found to be appropriate for the degradation of MO by NMPs. XPS and FT-IR analyses confirmed that NMPs were oxidized and corroded after the reaction. The results of the present study provide ample evidence of the removal of azo dye from wastewater via the application of NMPs under the sono-AFP.

\section{Acknowledgments}

The present research was conducted with the support of a research grant from Kwangwoon University in 2017. This work was also 
supported by the Korean Ministry of the Environment as part of the "GAIA project" (2014000550003).

\section{References}

1. Li P, Song Y, Wang S, Tao Z, Yu S, Liu Y. Enhanced decolorization of methyl orange using zero-valent copper nanoparticles under assistance of hydrodynamic cavitation. Ultrason. Sonochem. 2015;22:132-138.

2. Sha Y, Mathew I, Cui Q, et al. Rapid degradation of azo dye methyl orange using hollow cobalt nanoparticles. Chemosphere 2016;144:1530-1535.

3. Li Y, Zhang FS. Catalytic oxidation of Methyl Orange by an amorphous $\mathrm{FeOOH}$ catalyst developed from a high iron-containing fly ash. Chem. Eng. J. 2010;158:148-153.

4. Netpradit S, Towprayoon P, Thiravetyan S. Adsorption of three azo reactive dyes by metal hydroxide sludge: Effect of temperature, $\mathrm{pH}$, and electrolytes. J. Colloid Interface Sci. 2004;270:255-261.

5. Subramonian W, Wu TY. Effect of enhancers and inhibitors on photocatalytic sunlight treatment of methylene blue. Water Air Soil Pollut. 2014;225:1-15.

6. Teh CY, Budiman PM, Shak KPY, Wu TY. Recent advancement of coagulation-flocculation and its application in wastewater treatment. Ind. Eng. Chem. Res. 2016;55:4363-4389.

7. Nidheesh P, Gandhimathi R, Ramesh S. Degradation of dyes from aqueous solution by Fenton processes : A review. Environ. Sci. Pollut. Res. 2013;20:2099-2132.

8. Fang ZQ, Qiu XQ, Chen JH, Qiu XH. Degradation of metronidazole by nanoscale zero-valent metal prepared from steel pickling waste liquor. Appl. Catal. B-Environ. 2010;100:221-228.

9. Subramonian W, Wu TY, Chai SP. Photocatalytic degradation of industrial pulp and paper mill effluent using synthesized magnetic $\mathrm{Fe}_{2} \mathrm{O}_{3}-\mathrm{TiO}_{2}$ : Treatment efficiency and characterizations of reused photocatalyst. J. Environ. Manage. 2017;187:298-310.

10. Shih YH, Tso CP, Tung LY. Tung, Rapid degradation of methyl orange with nanoscale zerovalent iron particles. J. Environ. Eng. Manage. 2010;20:137-143.

11. Yuan N, Zhang G, Guo S, Wan Z. Enhanced ultrasoundassisted degradation of methyl orange and metronidazole by rectorite-supported nanoscale zero-valent iron. Ultrason. Sonochem. 2016;28:62-68.

12. Li H, Guo J, Yang L, Lan Y. Degradation of methyl orange by sodium persulfate activated with zero-valent zinc. Sep. Purif. Technol. 2014;132:168-173.

13. Singh J, Yang JK, Chang YY. Rapid degradation of phenol by ultrasound-dispersed nano-metallic particles (NMPs) in the presence of hydrogen peroxide: A possible mechanism for phenol degradation in water. J. Environ. Manage. 2016;175:60-66

14. Singh J, Yang JK, Chang YY. Synthesis of nano zero-valent metals from the leaching liquor of automobile shredder residue: A mechanism and potential applications for phenol degradation in water. Process Saf. Environ. 2016;102:204-213.

15. Chand R, Ince NH, Gogate PR, Bremner DH. Phenol degradation using 20, 300 and $520 \mathrm{kHz}$ ultrasonic reactors with hydrogen peroxide, ozone and zero valent metals. Sep. Purif. Technol. 2009;67:103-109.

16. Singh J, Lee BK. Pollution control and metal resource recovery for low grade automobile shredder residue: A mechanism, bioavailability and risk assessment. Waste Manage. 2015;38:271-283.

17. Singh J, Yang JK, Chang YY. Quantitative analysis and reduction of the eco-toxicity risk of heavy metals for the fine fraction of automobile shredder residue (ASR) using $\mathrm{H}_{2} \mathrm{O}_{2}$. Waste Manage. 2016;48:374-382.

18. Singh J, Lee BK. Hydrometallurgical recovery of heavy metals from low-grade automobile shredder residue (ASR): An application of an advanced Fenton process (AFP). J. Environ. Manage. 2015;161:1-10.

19. Teh CY, Wu TY, Juan JC. Facile sonochemical synthesis of N,Cl-codoped $\mathrm{TiO}_{2}$ : Synthesis effects, mechanism and photocatalytic performance. Catal. Today 2015;256:365-374.

20. Rasheed QJ, Pandian K, Muthukumar K. Treatment of petroleum refinery wastewater by ultrasound-dispersed nanoscale zero-valent iron particles. Ultrason. Sonochem. 2011;18: 1138-1142.

21. Zha SX, Cheng Y, Gao Y, Chen ZL, Megharaj M, Naidu R. Nanoscale zerovalent iron as a catalyst for heterogeneous Fenton oxidation of amoxicillin. Chem. Eng. J. 2014;255:141-148.

22. Haeberle J, Henkel K, Gargouri H, et al. Ellipsometry and XPS comparative studies of thermal and plasma enhanced atomic layer deposited $\mathrm{Al}_{2} \mathrm{O}_{3}$ films. Beilstein J. Nanotechnol. 2013;4:732-742.

23. Kim JH, Cho S, Bae TS, Lee YS. Enzyme biosensor based on an N-doped activated carbon fiber electrode prepared by a thermal solid-state reaction. Sens. Actuators B-Chem 2014;197:20-27.

24. Biesinger MC, Lau LWM, Gerson AR, Smart RSC. Resolving surface chemical states in XPS analysis of first row transition metals, oxides and hydroxides: Sc, Ti, V, Cu and Zn. Appl. Surf. Sci. 2010;257:887-898.

25. Dong G, Ai Z, Zhang L. Total aerobic destruction of azo contaminants with nanoscale zero-valent copper at neutral $\mathrm{pH}$ : Promotion effect of in-situ generated carbon center radicals. Water Res. 2014;66:22-30.

26. Ilton ES, Post JE, Heaney PJ, Ling FT, Kerisit SN. XPS determination of Mn oxidation states in Mn (hydr) oxides. Appl. Surf. Sci. 2016;366:475-485.

27. Morozov IG, Belousova OV, Ortega D, Mafina MK, Kuznetcov MV. Structural, optical, XPS and magnetic properties of Zn particles capped by $\mathrm{ZnO}$ nanoparticles. J. Alloy. Compd. 2015;633:237-245.

28. Xu L, Wang J. A heterogeneous Fenton-like system with nanoparticulate zero-valent iron for removal of 4-chloro-3-methyl phenol. J. Hazard. Mater. 2011;186:256-264.

29. Babuponnusami A, Muthukumar K. Removal of phenol by heterogeneous photo electro Fenton-like process using nano-zero valent iron. Sep. Purif. Technol. 2012;98:130-135.

30. Houa L, Wang L, Royer S, Zhang H. Ultrasound-assisted heterogeneous Fenton-like degradation of tetracycline over a magnetite catalyst. J. Hazard. Mater. 2016;302:458-467. 
31. Rao Y, Yang H, Xue D, Guo Y, Qi F, Ma J. Sonolytic and sonophotolytic degradation of Carbamazepine: Kinetic and mechanisms. Ultrason. Sonochem. 2016;32:371-379.

32. Weiping X, Yan Q, Dingmin L, Dan S, Dewen H. Degradation of m-xylene solution using ultrasonic irradiation. Ultrason.
Sonochem. 2011;18:1077-1081.

33. Cheng Z, Fu F, Pang Y, Tang B, Lu J. Removal of phenol by acid-washed zero-valent aluminium in the presence of $\mathrm{H}_{2} \mathrm{O}_{2}$. Chem. Eng. J. 2015;260:284-290. 